\title{
ArcheoSciences
}

Revue d'archéométrie

\section{Les ivoires d'Arslan Tash (Syrie) d'après une étude de la collection du Musée du Louvre : mise en œuvre du matériau, traces de polychromie et de dorure, état de conservation}

Study of Arslan Tash Ivories (Syria) from the Louvre Collection: Making of the Material, Polychrome and Gilding Traces, Conservation State

\section{Elisabeth Fontan et Ina Reiche}

\section{(2) OpenEdition}

\section{Journals}

\section{Édition électronique}

URL : https://journals.openedition.org/archeosciences/3210

DOI : 10.4000/archeosciences.3210

ISBN : 978-2-7535-1849-0

ISSN : 2104-3728

Éditeur

Presses universitaires de Rennes

Édition imprimée

Date de publication : 30 avril 2011

Pagination : 283-295

ISBN : 978-2-7535-1847-6

ISSN : 1960-1360

Référence électronique

Elisabeth Fontan et Ina Reiche, « Les ivoires d'Arslan Tash (Syrie) d'après une étude de la collection du Musée du Louvre : mise en œuvre du matériau, traces de polychromie et de dorure, état de conservation », ArcheoSciences [En ligne], 35 | 2011, mis en ligne le 30 avril 2013, consulté le 28 février 2022. URL : http://journals.openedition.org/archeosciences/3210 ; DOI : https://doi.org/10.4000/ archeosciences.3210 


\title{
Les ivoires d'Arslan Tash (Syrie) d'après une étude de la collection du Musée du Louvre : mise en œuvre du matériau, traces de polychromie et de dorure, état de conservation
}

\author{
Study of Arslan Tash Ivories (Syria) from the Louvre Collection: \\ Making of the Material, Polychrome and Gilding Traces, Conservation State
}

\author{
Elisabeth FonTan* et Ina ReICHE **
}

\begin{abstract}
Résumé : Les ivoires d'Arslan Tash constituent un ensemble exceptionnel découvert en 1928 sur un site de Syrie du Nord à quelques kilomètres de la frontière turque, à l'est de l'Euphrate, par une mission archéologique du Louvre. Arslan Tash, l'ancienne Hadatu, a été le siège d'une capitale provinciale à l'époque de l'empire néo-assyrien (IX-VII ${ }^{\mathrm{e}}$ siècles avant J.-C.).

Lors de deux campagnes de fouilles, un bâtiment voisin du palais a été mis au jour où furent découverts dans la pièce 14 ces ivoires sculptés qui avaient décoré un mobilier d'apparat. Ils ont été considérés alors comme le plus bel ensemble d'ivoires phéniciens connus.

Selon la réglementation en vigueur à l'époque du mandat français, ces ivoires ont été partagés entre le Musée national d'Alep et le Musée du Louvre. Les œuvres attribuées au Louvre y arrivèrent en 1929. Certaines furent confiées pour restauration à la maison André à Paris.

D’autres ivoires très proches par le style ont été acquis sur le marché de l'art après la seconde guerre mondiale par différents musées. Une nouvelle publication de l'ensemble de ce matériel est en préparation qui comprend des études historiographique, stylistique, technique et épigraphique. Elle est menée en collaboration par une équipe composée de : Giorgio Affanni, Annie Caubet, Serena Cecchini, Élisabeth Fontan, François Poplin et Maria Giulia Amadasi.

Récemment a été entrepris un programme de recherche visant à étudier plus particulièrement la mise en œuvre du matériau, les questions de polychromie et de dorure ainsi que l'état de conservation des pièces. Seize œuvres, sélectionnées dans le corpus des ivoires d'Arslan Tash de la collection du Musée du Louvre, ont été soumises à différentes analyses non-destructives par microPIXE/PIGE, FT-IR, Raman ainsi que par radiographie et par tomographie X. Les résultats de ces analyses fournissent des informations nouvelles sur les traces de sédiment ainsi que de polychromie et, particulièrement sur la nature des taches pourpres; ils montrent l'intérêt de mener une étude plus détaillée de l'ensemble des ivoires d'Arslan Tash en collaboration avec d'autres musées.
\end{abstract}

\begin{abstract}
The Arslan Tash ivories form an exceptional set discovered in 1928 by a mission of the Louvre museum on a site in Northern Syria some kilometers from the Turkish border, in the East of the Euphrates. Arslan Tash, known under the ancient name Hadatu, was the place of a provincial capital during the time of the Neo-Assyrian Empire (9-7th $c . B C$ ). In the course of two excavation campaigns, a neighboring building of the palace was unearthed where pieces of carved ivory were recovered in room 14. These ivories decorated pieces of luxury furniture. It was then considered as the most beautiful known set of Phoenician ivories.

According to the effective legislation of the time of the French Mandate over Syria and the Lebanon, these ivories were shared between the National museum of Aleppo and the Louvre museum. The works of art allocated to the Louvre arrived in 1929. Some were given to the Maison André in Paris for conservation work.
\end{abstract}

* Département des Antiquités Orientales, Musée du Louvre - Pavillon Mollien, 75001 Paris. (elisabeth.fontan@louvre.fr)

** UMR 171 CNRS, Laboratoire du Centre de Recherche et de Restauration des Musées de France, Palais du Louvre, 14 quai François-Mitterrand, 75001 Paris, France. (ina.reiche@culture.gouv.fr) 
Other ivories very close in style were acquired from the art market after the Second World War by different museums. A new publication of the whole material is in preparation which comprises historiographical, stylistic, technical and epigraphical studies. They are performed in collaboration with a team composed of: Giorgio Affanni, Annie Caubet, Serena Cecchini, Elisabeth Fontan, François Poplin and Maria Giulia Amadasi.

Recently a research programme was undertaken aiming at studying particularly the object fabrication, the questions of polychromy and gilding and the evaluation of the conservation state of the pieces. Sixteen pieces selected within the corpus of Arslan Tash ivories from the Louvre museum collection, were analyzed non-destructively by microPIXE/PIGE, FT-IR, Raman spectroscopy and X-ray radiography and tomography. The results of these investigations provide new insights into the conservation state of the ivories, the nature of sediment as well as polychrome traces, and particularly of the purple zones on the ivories; they show the importance of a more precise materials characterization of the Arslan Tash ivories in collaboration with other museums.

Mots clés : ivoire, Arslan Tash, phénicien, manufacture, polychromie, dorure, conservation.

Keywords: ivory, Arslan Tash, Phoenician, making, polychromy, gilding, conservation

À Thierry Borel (1958-2011)

\section{INTRODUCTION}

Les ivoires présentés ici constituent un ensemble exceptionnel découvert sur le site d'Arslan Tash, nom qui signifie " la pierre au lion ", au nord de la Syrie à quelques kilomètres de la frontière turque, à l'est de l'Euphrate.

Les fouilles ont été menées sous la direction de François Thureau-Dangin, conservateur en chef du département des Antiquités Orientales du musée du Louvre, en 1928 au cours de deux campagnes.

C'est dans les quinze derniers jours de la première campagne, après le départ de Thureau-Dangin, qu'a eu lieu la découverte du lot le plus important des fameux ivoires par le père dominicain Augustin Barrois, membre de l'École biblique et archéologique française de Jérusalem. Les ivoires trouvés lors de la deuxième campagne sont moins nombreux et généralement en mauvais état de conservation.

Les fouilles ont notamment mis au jour un palais néoassyrien du I ${ }^{\text {er }}$ millénaire avant J.-C. et un autre bâtiment plus ancien appelé en raison de la découverte " le Bâtiment aux ivoires " qui était sans doute un autre palais. Arslan Tash, nommée Hadatu dans l'antiquité, était, comme le site voisin de Tell Ahmar/Til Barsip, une capitale provinciale de l'empire assyrien aux $\mathrm{IX}^{\mathrm{e}}$-VIIII ${ }^{\mathrm{e}}$ siècles. Les ivoires qualifiés traditionnellement de phéniciens constituaient le décor d'un mobilier, associés à des éléments de verre coloré. Les fouilleurs ont retrouvé les traces d'un ou peut être deux lits ou bien d'un lit et d'un trône (fig. 1).

La publication de la fouille est parue très rapidement, dès 1931. Cent seize ivoires y sont publiés. Ils ont été partagés, selon la réglementation en vigueur à l'époque du mandat français, entre le musée d'Alep ( $\mathrm{n}: 71)$ et le Louvre ( $\mathrm{n}: 46)$.

Un certain nombre d'ivoires qui figurent dans la publication ne sont répertoriés ni à Alep ni au Louvre. En revanche quelques œuvres exposées dans les vitrines d'Alep ne sont pas mentionnées dans cette publication. De plus, récemment sont apparues six plaquettes conservées à l'École biblique et archéologique française de Jérusalem qui avaient " échappé " au partage officiel. Elles ont été rapportées par le Père Barrois et ont été immédiatement inscrites sur le registre d'inventaire de l'École en juillet 1928 sans aucune indication de provenance.

Cependant un nombre important d'ivoires attribués d'un point de vue stylistique à Arslan Tash se trouvent dans plusieurs autres musées où ils sont arrivés par la voie du commerce de l'art. Ils ont tous été acquis d'Élie Borowski, marchand établi à Bâle, qui les aurait achetés en 1955 à un confrère parisien spécialisé dans l'art perse. Le Metropolitan Museum de New York a ainsi acquis dix sept œuvres en 1957, le musée de Hambourg une femme à la fenêtre en 1966, le Badisches Landesmuseum de Karlsruhe 41 œuvres et une centaine de fragments entre 1970 et 1972. Enfin E. Borowski a déposé, en 1992, 21 pièces au Bible Lands Museum de Jérusalem, crée pour abriter sa collection.

L’origine de ces œuvres n'est pas assurée. Elles proviennent soit de fouilles clandestines opérées entres les deux campagnes ou à la fin de la seconde, car Thureau-Dangin indique qu'il a laissé sur place des fragments impossibles à dégager, soit d'un vol dans un local de stockage en Syrie.

Â l'occasion de la nouvelle présentation des ivoires d'Arslan Tash du Louvre dans les salles de l'aile Richelieu en 1993, une campagne de restauration de l'ensemble de la collection a été entreprise accompagnée d'une étude scientifique. 
Figure 1 : Découverte du cadre de lit et des premières plaquettes d'ivoire dans la salle 14 du "Bâtiment aux ivoires ", Thureau-Dangin et al., 1931, pl. XVIII, 1.

Figure 1: Discovery of the bed frame and first ivory plaquettes in the room 14 of the "ivory building", Thureau-Dangin et al., 1931, pl. XVIII, 1.

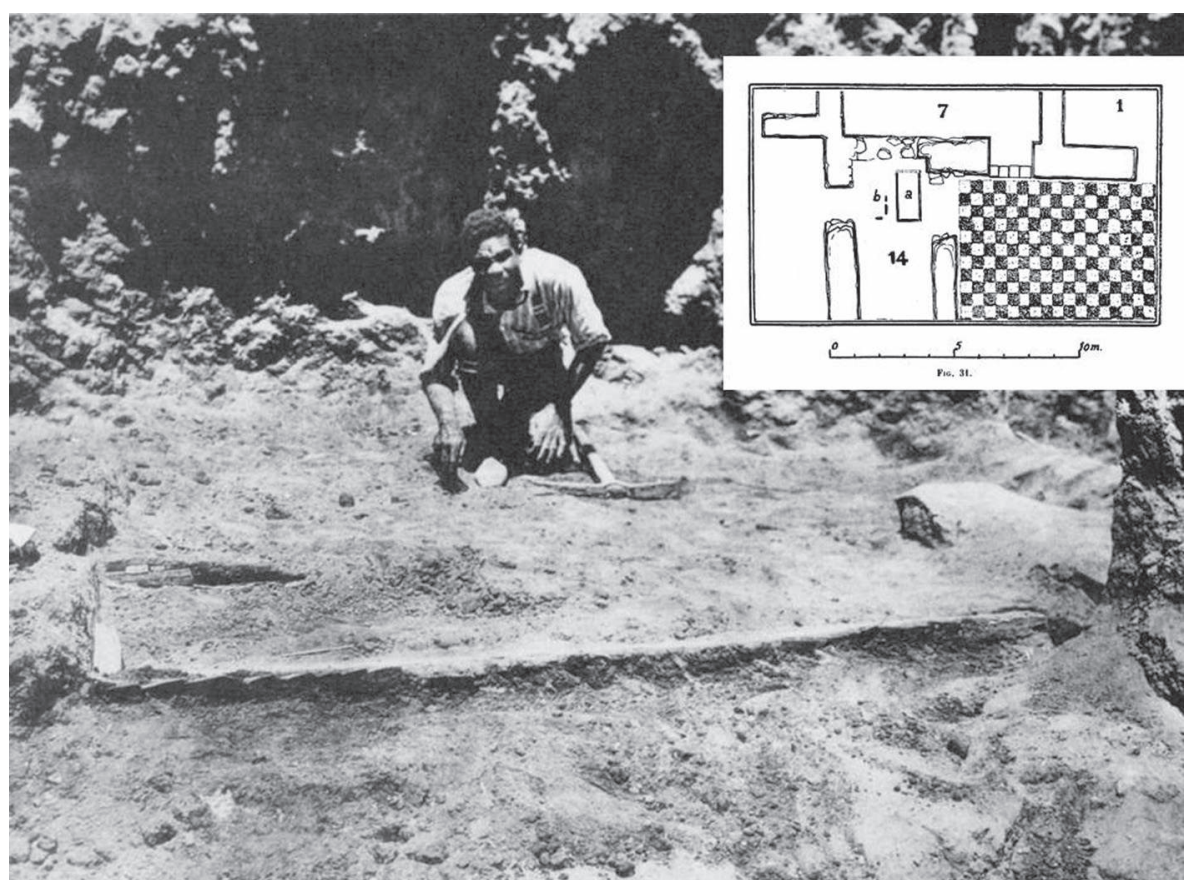

Parallèlement dans le cadre de la coopération du Louvre avec la Direction générale des Antiquités et des Musées de Syrie, Annie Caubet avait lancé en 2003 un programme d'étude et restauration des ivoires des musées syriens. À la suite de quatre missions à Alep entre 2005 et 2009, l'étude de la collection d'Arslan Tash est terminée et les restaurations, confiées à Juliette Lévy qui a restauré les ivoires du Louvre et Marie-Emmanuelle Meyohas, sont en cours.

Pour compléter ces travaux il était indispensable d'examiner les ivoires conservés à l'Ecole biblique afin d'avoir une vision de l'ensemble sorti de la fouille, puis ceux du Metropolitan Museum, du Badisches Landesmuseum, du Museum für Kunst und Gewerbe de Hambourg et du Bible Lands Museum.

Par ailleurs, l'université de Bologne sous la direction du Professeur Serena Maria Cecchini a entrepris une nouvelle mission d'étude sur le terrain au cours de trois courtes campagnes en 2007, 2008 et 2009 pour tenter de préciser la stratigraphique et la chronologie. En effet, au cours des fouilles anciennes aucune céramique ou tesson n'a été recueilli. Le site n'ayant pas été protégé après la fouille Thureau-Dangin, qui a duré à peine cinq mois, a été entièrement recouvert par les constructions modernes. Il y a donc très peu d'espaces libres pour pratiquer des sondages sur l'emplacement présumé de la salle $14 \mathrm{du}$ "bâtiment aux ivoires ".

À la suite de toutes ces recherches, une nouvelle publication de l'ensemble des ivoires d'Arslan Tash est en préparation. Cette publication collective sera réalisée en collaboration par le Musée du Louvre, l'université de Bologne et la Direction générale des Antiquités et des Musées de Syrie. Elle comprendra une étude historiographique, une étude stylistique, une étude technique et une étude épigraphique. Elle est menée par une équipe composée de : Giorgio Affanni, Annie Caubet, Serena Cecchini, Élisabeth Fontan, François Poplin et Maria Giulia Amadasi.

Ce projet s'inscrit dans le cadre plus large des nouvelles études sur les ivoires du ${ }^{\text {er }}$ millénaire menées par Georgina Herrmann pour Nimrud et par Claudia Suter pour Samarie.

Pour répondre aux questions que nous nous posions à la suite de ces observations, nous avons fait appel au C2RMF. En 2006 deux œuvres ont été analysées [2]. Récemment a été mis en place un programme de recherche visant à étudier plus particulièrement la mise en œuvre du matériau, les questions de polychromie (fig. 2) et de dorure (fig. 3), ainsi que l'état de conservation des pièces et la nature des restaurations effectuées. Le but de ces recherches est de finement déterminer les caractéristiques des ivoires afin de disposer d'éléments de comparaison pour les ivoires d'origine incertaine.

\section{Matériel}

Seize oeuvres en ivoire sculpté, peint et/ou doré, provenant du site d'Arslan Tash en Syrie (viII ${ }^{\mathrm{e}}$ s. av. J.-C.) et conservés au Département des Antiquités Orientales du Musée du Louvre ont été sélectionnés pour les analyses (fig. 4, tableau 1). Elles ont été soumises à différentes analyses 

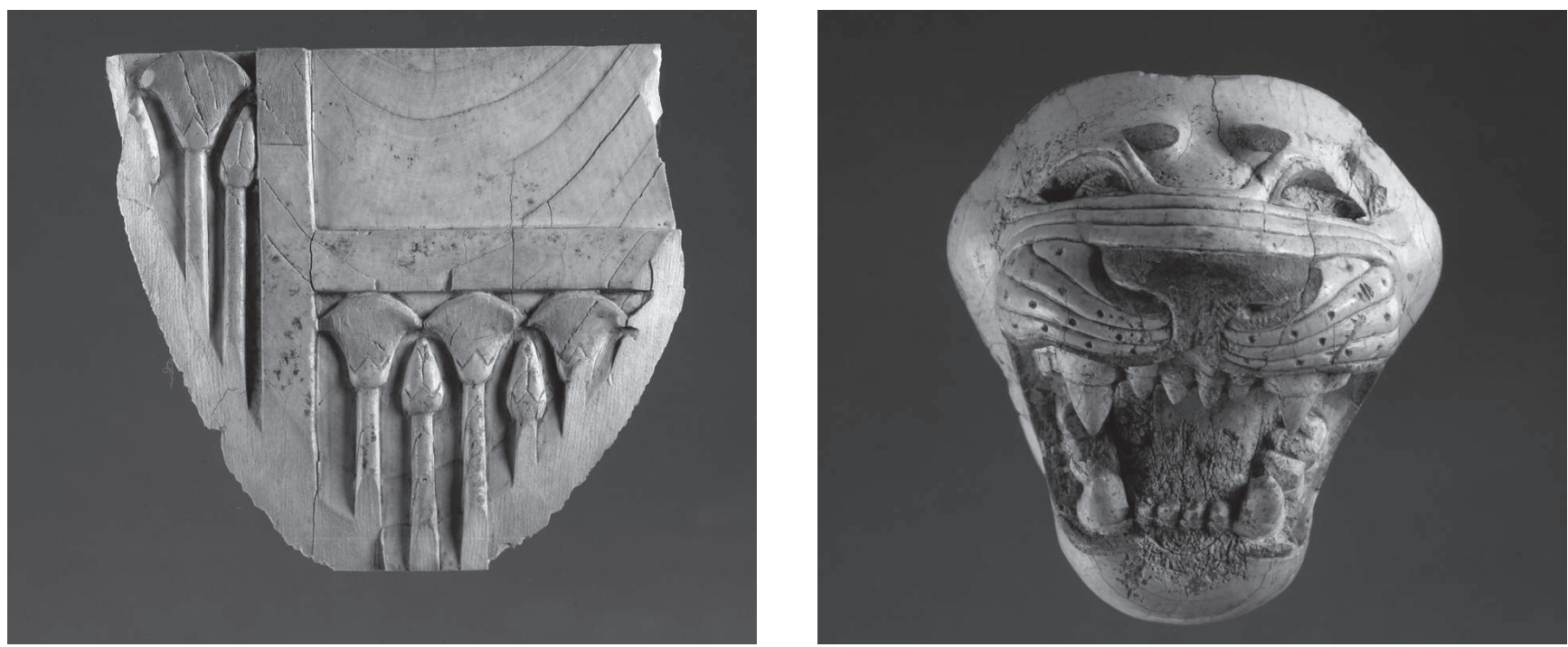

Figure 2 : (Voir planche couleur) Plaquette aux papyrus (AO 11480) et tête de lion (AO 11490) présentant des traces de polychromie, (C) Musée du Louvre/R. Chipault.

Figure 2: (See colour plate) Papyrus plaquette (AO 11480) and lion head (AO 11490) showing traces of polychromy, (C) Musée du Louvre/R. Chipault.

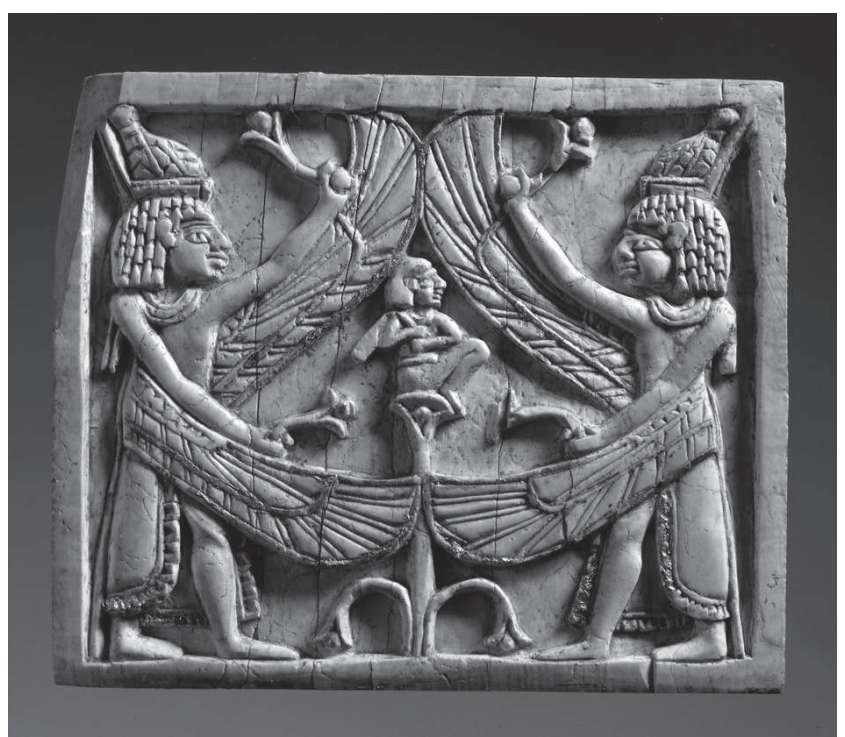

Figure 3 : (Voir planche couleur) Plaquettes présentant des restes de feuille d'or (à gauche AO 11465, non analysée) ou des traces de couleur violette (AO 11469) en indiquant la présence à l'origine, (C) Musée du Louvre/R. Chipault.

Figure 3: (See colour plate) Plaquettes showing remains of gilding (on left hand side $A O 11465$, not analyzed) or purple color traces ( $A O$ 11469) indicating their presence originally, (C) Musée du Louvre/R. Chipault.

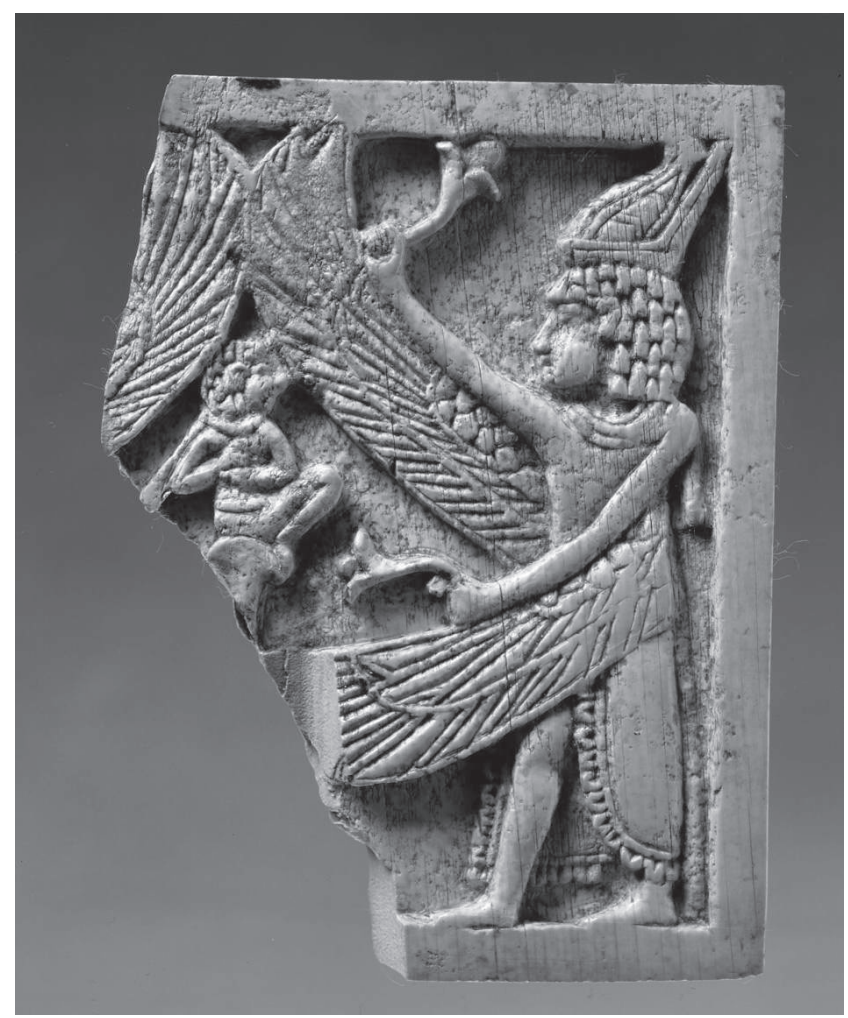

ArcheoSciences, revue d'archéométrie, 35, 2011, p. 283-295 


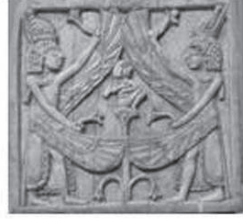

AO 11466

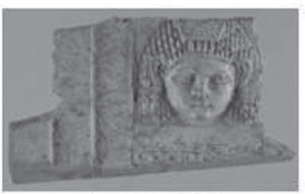

AO 11464

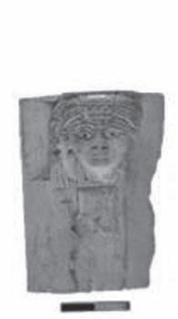

AO 11462

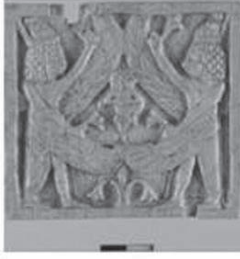

AO 11467

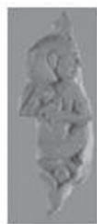

AO 11494

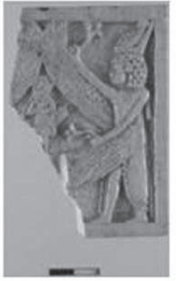

AO 11469

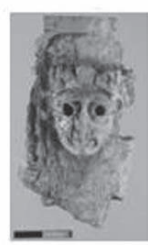

AO 11463
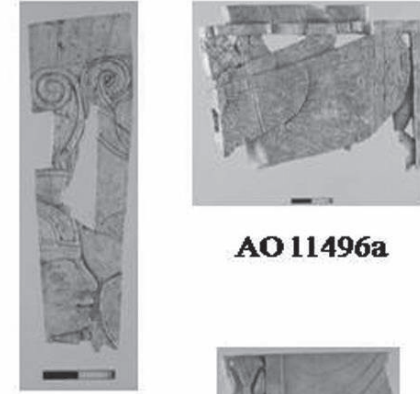

AO11486

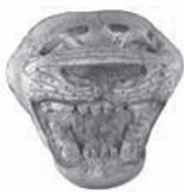

AO 11490
AO 11496a

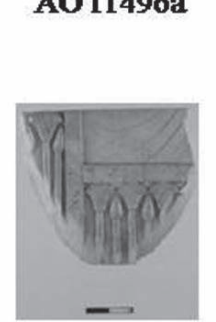

AO 11480

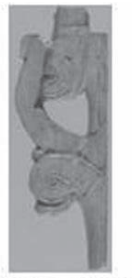

AO 11496b

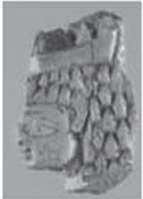

AO 11495

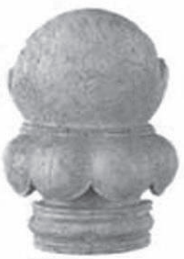

AO 11484

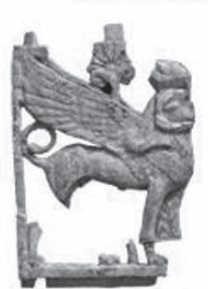

AO 11497

Figure 4 : Les seize ivoires analysés au C2RMF.

Figure 4: Sixteen ivories analyzed at the C2RMF.

\begin{tabular}{|c|c|c|c|c|}
\hline $\mathrm{n}^{\circ}$ inventaire & Description & Lieu & $\mathrm{n}^{\circ}$ C2RMF & $\mathrm{n}^{\circ}$ Laboratoire \\
\hline AO 11462 & Dame à la fenêtre, fragment & Réserve & C2RMF64594 & AI_AT_Louv1 \\
\hline AO 11463 & Dame à la fenêtre, fragment & Réserve & C2RMF64600 & AI_AT_Louv2 \\
\hline AO 11464 & Dame à la fenêtre, fragment & Réserve & C2RMF64599 & AI_AT_Louv3 \\
\hline AO 11466 & Naissance d'Horus & Réserve & C2RMF64601 & AI_AT_Louv14 \\
\hline AO 11467 & Naissance d'Horus & Exposé & C2RMF61024 & AI_AT_Louv4 \\
\hline AO 11469 & Naissance d'Horus, fragment & Réserve & C2RMF64596 & AI_AT_Louv5 \\
\hline AO 11480 & Plaquette aux papyrus, fragment & Exposé & C2RMF61484 & AI_AT_Louv6 \\
\hline AO 11484 & Colonnette (Pied de meuble) & Exposé & C2RMF61852 & AI_AT_Louv7 \\
\hline AO 11486 & Plaquette avec une tête de sphinx, fragment & Réserve & C2RMF64602 & AI_AT_Louv8 \\
\hline AO 11490 & Tête de lion en haut relief & Exposé & C2RMF61854 & AI_AT_Louv9 \\
\hline AO 11494 & Naissance d'Horus, enfant & Réserve & C2RMF64603 & AI_AT_Louv10 \\
\hline AO 11495 & Naissance d'Horus, tête & Réserve & C2RMF64604 & AI_AT_Louv11 \\
\hline AO $11496 \mathrm{a}$ & Sphinx, fragment aile & Exposé & C2RMF63906 & AI_AT_Louv12 \\
\hline AO $11496 \mathrm{~b}$ & Sphinx, fragment palmette & Réserve & C2RMF64598 & AI_AT_Louv13 \\
\hline AO 11497 & Sphinx, fragment & Exposé & C2RMF61658 & AI_AT_Louv15 \\
\hline AO 11452 & Vache léchant son veau & Exposé & C2RMF61836 & AI_AT_Louv16 \\
\hline
\end{tabular}

Tableau 1 : Description et désignation des objets en ivoire d'Arslan Tash étudiés.

Table 1: Description and names of the studied ivory objects of Arslan Tash.

non-destructives, à l'exception de deux micro échantillons prélevés sur les pièces AO 11463 et 11494 sur lesquelles des observations et analyses en microscopie électronique à balayage (MEB) et en spectrométrie infrarouge à transformée de Fourrier (IR-TF) ont été possibles. Ceci permet une meilleure évaluation de l'état de conservation de ces objets. 


\section{Méthodes}

\section{Analyse élémentaire par émission de rayons $\mathrm{X}$ et gamma induits par un microfaisceau de protons (microPIXE/PIGE)}

Des analyses de la composition chimique de l'ivoire ont été réalisées avec la méthode microPIXE à l'accélérateur Grand Louvre d'Analyse Élémentaire (AGLAE) installé au C2RMF à Paris. Les mesures ont été effectuées avec des protons de $3 \mathrm{MeV}$, un courant d'environ $1 \mathrm{nA}$ et un faisceau incident extrait à l'hélium d'un diamètre de $50 \mu \mathrm{m}^{2}$ [3].

\section{Analyse structurale}

Pour préciser la nature des matériaux de polychromie, des analyses en spectroscopie Raman ont été effectuées sur les objets en mode confocal à l'aide d'une sortie horizontale du micro-spectromètre LabRam Infinity (Jobin-Yvon) en utilisant un laser d'excitation avec une longueur d'onde de 632,5 nm. De plus, des expériences de microdiffraction de rayons $\mathrm{X}$ (microDRX) ont été menées avec un tube Rigaku SC Micromax 002 (anode de $\mathrm{Cu}$, tension de $45 \mathrm{kV}$, courant de $660 \mu \mathrm{A}$, puissance ca. $30 \mathrm{~W}$, optique de type KirkpatrickBaez) équipé d'un collimateur formant un faisceau incident quasi-parallèle avec un diamètre de $200 \mu \mathrm{m}$ et un détecteur 2D R-Axis IV+ [4].

Les analyses en IRTF ont été faites sur micro-prélèvement à l'aide d'un spectromètre Spectrum 2000 (Perkin Elmer) en mode de transmission en employant une cellule de diamant. Ceci a l'avantage de n'utiliser que très peu de matière. Les spectres ont été enregistrés dans une gamme allant de 4000 à $400 \mathrm{~cm}^{-1}$ avec une résolution de $4 \mathrm{~cm}^{-1}$. Quatre scans ont été accumulés pour chaque spectre. L'évaluation des données a été réalisée grâce au logiciel GRAMS. Après une correction de la ligne de base, les intensités des bandes d'absorption ont été déterminées pour calculer des paramètres spécifiques de l'état de conservation de l'ivoire. L'état de la phase minérale peut être décrite par un indice de cristallinité nommé infrared splitting factor (IRSF) basé sur l'intensité des bandes de vibration des phosphates à 565 et $605 \mathrm{~cm}^{-1}$ et la teneur en carbonate est représentée par le rapport d'intensité de la bande carbonate principale à $1415 \mathrm{~cm}^{-1}$ et de la bande phosphate à $1035 \mathrm{~cm}^{-1}[5,6]$.

\section{Observations morphologiques par radio- et tomographie X sur objet et par MEB sur prélèvement}

Les clichés de radiographie et de tomographie $\mathrm{X}$ ont été pris avec le dispositif disponible au C2RMF [7]. Les observations microscopiques ont été faites avec un MEB XL 30 CP (Philips) équipé d'un détecteur $\mathrm{X}$ dispersive en énergie Link Isis 300. La tension d'accélération utilisée est de $20 \mathrm{kV}$ avec un courant d'environ $100 \mu \mathrm{A}$. Le fragment a été analysé sans préparation particulière pour le conserver pour d'autres analyses.

\section{Résultats ET DISCUSSION}

\section{État de conservation de l'ivoire}

\section{Observation macroscopique}

À l'œil nu, les ivoires d'Arslan Tash sont bien conservés et très clairs. Leur cohésion est satisfaisante et permet la manipulation des pièces. Néanmoins, quelques signes d'altération sont visibles : des parties manquantes, une surface émoussée, des fissures et des traces de polychromie ainsi que de consolidants. Il faut aussi remarquer quelques variations des états de conservation à l'intérieur du corpus : l'objet AO 11469 semble être le mieux préservé, tandis que l'objet AO 11463 le plus altéré. De plus, sur une même pièce, l'état de surface peut être hétérogène. Par exemple, le côté gauche de la plaquette $\mathrm{AO} 11466$ est très fissuré en surface, alors que le côté droit est beaucoup plus lisse.

\section{Observation microscopique}

Un micro-prélèvement effectué sur l'objet AO 11463 présentant un état d'altération relativement avancé a permis d'observer une paillette d'ivoire au MEB afin d'appréhender sa structure microscopique (fig. 5). Ainsi, l'ivoire apparaît très fissuré, fragmenté et friable ; il a en partie perdu sa cohésion. Il est cependant encore possible de distinguer les tubules caractéristiques de l'ivoire, montrant qu'il a quand même en partie conservé sa structure microscopique.

\section{Analyse élémentaire par microPIXE/PIGE}

Pour déterminer la composition chimique de l'ivoire par microPIXE, nous avons choisi des zones "vierges", comme des cassures récentes, où le risque était le plus limité possible de détecter les éléments chimiques caractéristiques des traitements de surface, restaurations, ou restes de sédiments en plus de ceux de l'ivoire. La représentation graphique des 


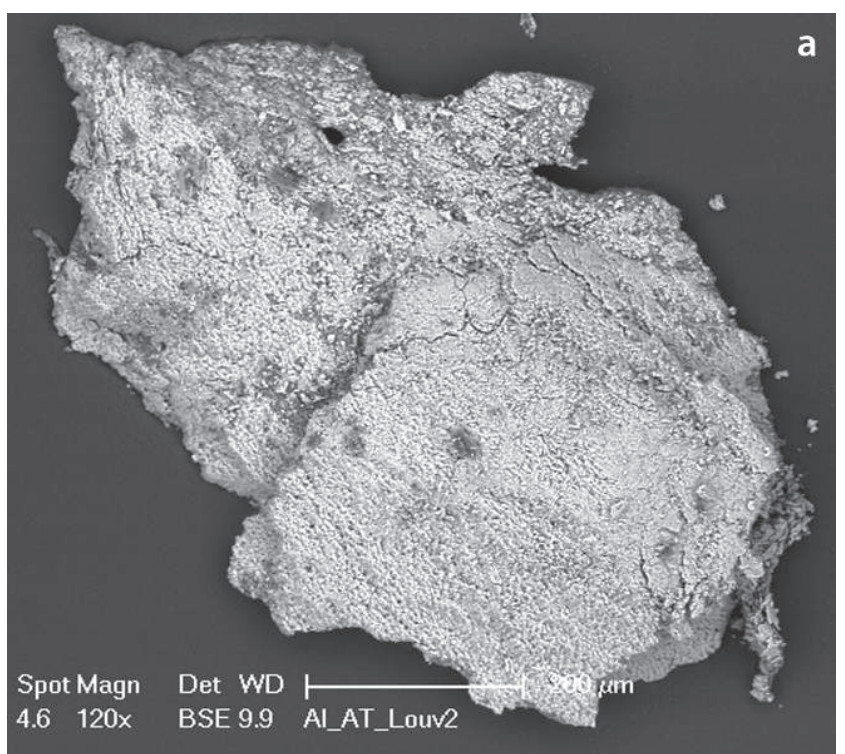

Figure 5 : Micrographies électroniques sur le prélèvement de l'objet AO 11463, a) vue globale, b) effritement et c) micrographie électronique de $20 \times 20 \mu \mathrm{m}^{2}$ montrant des tubules caractéristiques de l'ivoire.

Figure 5: Electron micrographs of the sample of the object $A O 11463$, a) global view, b) flaking and c) electron micrograph of $20 \times 20 \mathrm{\mu m}^{2}$ showing the characteristic ivory tubules.

rapports $\mathrm{CaO} / \mathrm{P}_{2} \mathrm{O}_{5}$ sur $\mathrm{MgO} / \mathrm{P}_{2} \mathrm{O}_{5}$, constituants chimiques majeurs et mineurs caractéristiques de l'ivoire, montre bien des états de conservation des différents objets altérés par rapport à l'ivoire moderne (fig. 6) dont le rapport $\mathrm{MgO} / \mathrm{P}_{2} \mathrm{O}_{5}$ se situe autour de 0,1 et celui de $\mathrm{CaO} / \mathrm{P}_{2} \mathrm{O}_{5}$ vers 1,1 . S' il existe un « noyau » de dix objets présentant un état de conservation proche, les quatre autres analysés se distinguent, avec des rapports relativement dispersés. Au vu de l'historique de ces objets, beaucoup de paramètres peuvent avoir joué sur la composition chimique et par conséquent expliquer les variations : la polychromie de surface, les conditions d'enfouissement sur le site archéologique, les conditions climatiques lors des fouilles, les restaurations subies, et les conditions climatiques pendant les expositions, pour ne citer que les plus importants.

\section{Analyses structurale de l'ivoire par IRTF}

Des micro-prélèvements sur deux objets ont permis des mesures en IRTF sur de l'ivoire représentatif de l'état de conservation de la majorité des objets (objet AO 11494), ainsi que sur un ivoire présentant un état de conservation différent (objet AO 11463).
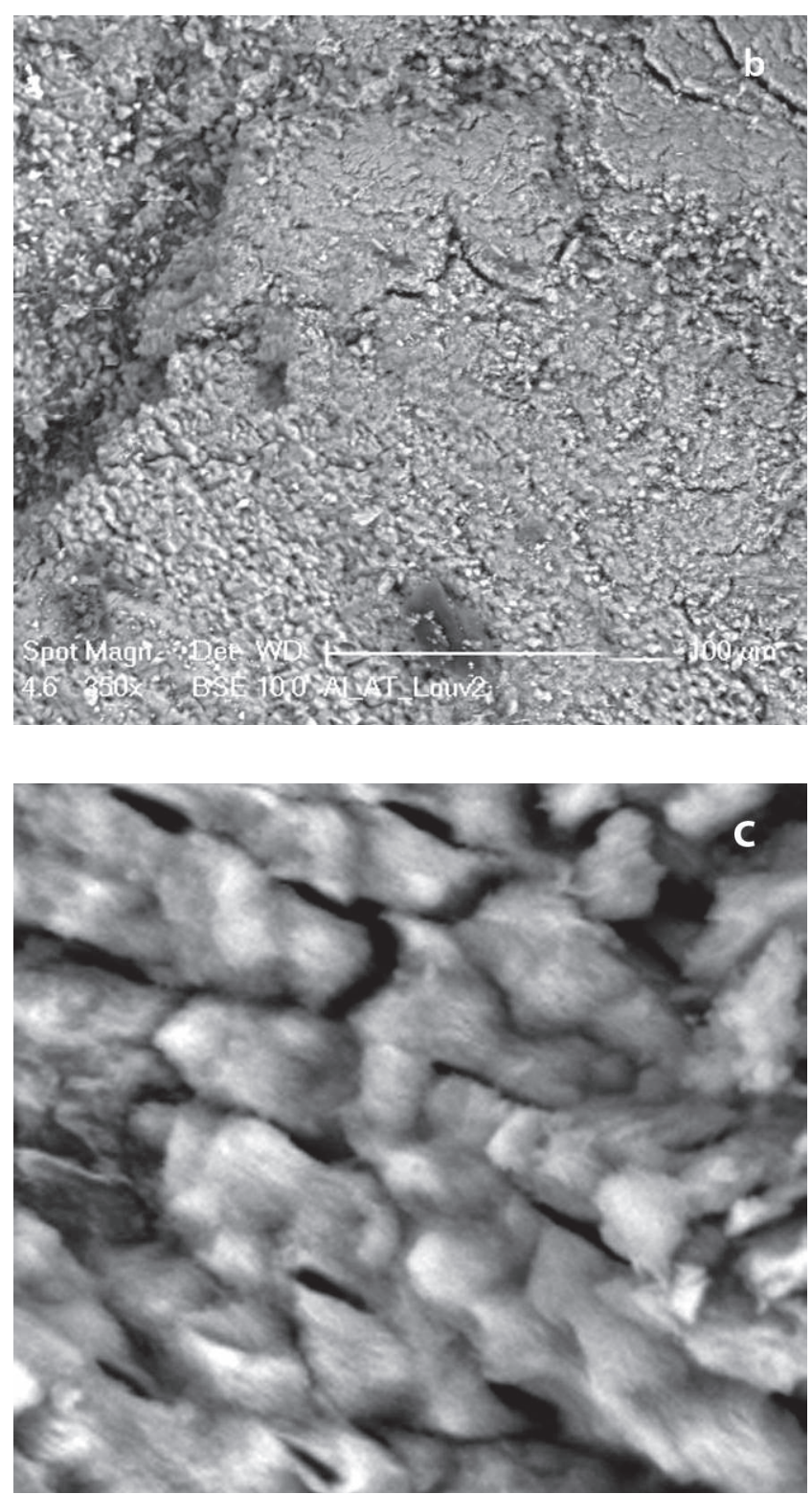

La comparaison des spectres IRTF obtenus pour les objets d'Arslan Tash avec celui d'une référence d'ivoire moderne d'éléphant montre que toutes les bandes spécifiques du collagène, bien visibles pour l'ivoire moderne, ne sont plus présentes sur les spectres obtenus pour les objets d'Arslan Tash. Ainsi, la quantité de collagène restant dans ces ivoires est si faible qu'elle n'est pas détectable par spectrométrie IRTF. Il n'y a donc plus de collagène dans les objets $A O 11463$ et 11494. Il parait possible d'extrapoler qu'il en est de même pour l'ensemble de la collection. Cela devrait entrainer une grande décohésion du matériau. Cependant les objets, bien que fragiles, restent en forme et sont manipulables. Il s'agit probablement de l'effet des restaurations successives permettant ainsi leur cohésion. 


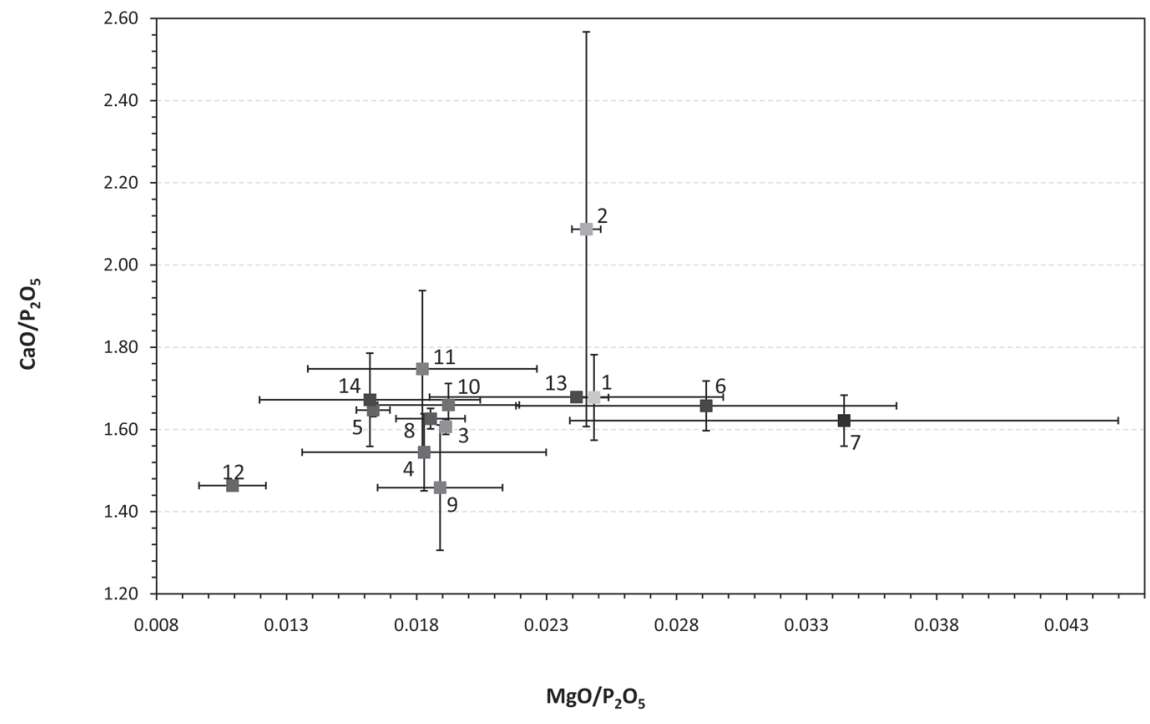

Figure 6 : Rapports $\mathrm{CaO} / \mathrm{MgO}$ normalisés de quatorze objets en ivoire du département des Antiquités orientales du Louvre, calculés à partir des résultats microPIXE. La valeur moyenne de l'ivoire moderne se situe en dehors de ce graphique $\left(\mathrm{MgO} / \mathrm{P}_{2} \mathrm{O}_{5}\right.$ de 0,1 et $\mathrm{CaO} /$ $\mathrm{P}_{2} \mathrm{O}_{5}$ vers 1,1$)$ et n'est pas représentée pour pouvoir mieux comparer les ivoires d'Arslan Tash entre eux.

Figure 6: Normaliezd CaO/MgO ratios of forteen ivory objects of the Oriental Antiquities department of the Louvre, calculted from microPIXE results. The mean value of modern ivory is situated outside of the graph with a $\mathrm{MgO} / \mathrm{P}_{2} \mathrm{O}_{5}$ ratio of 0,1 and a $\mathrm{CaO} / \mathrm{P}_{2} \mathrm{O}_{5}$ ratio of about 1,1 and is not shown here for better comparison among the Arslan Tash pieces.

\begin{tabular}{|l|c|c|c|}
\hline & Ivoire Moderne & AO 11463 & AO 11494 \\
\hline Cristallinité & $1,03 \pm 0,40$ & $2,00 \pm 0,01$ & $2,40 \pm 0,20$ \\
\hline Taux de carbonates & $0,38 \pm 0,08$ & $0,53 \pm 0,04$ & $0,58 \pm 0,29$ \\
\hline
\end{tabular}

Tableau 2 : Coefficients structuraux de l'ivoire moderne d'éléphant et des prélèvements d'objets d'Arslan Tash.

Table 2: Structural coefficients of modern elephant ivory and of the samples taken from Arslan Tash objects.

Deux coefficients caractéristiques de la phase minérale ont été calculés à partir des spectres IRTF, en faisant une moyenne sur deux ou trois spectres respectivement (tableau 2). Les deux objets d'Arslan Tash présentent un fort taux de carbonates, probablement d'origine exogène, ainsi qu'une cristallinité bien plus importante que celle de l'ivoire moderne et sont donc altérés au niveau de leur phase minérale.

\section{Identification des restes de dorure, de polychromie, de sédiments et d'anciennes restaurations}

Tous les ivoires d'Arslan Tash présentent des traitements de surface soit des polychromies ou des restes de sédiments. Ils ont également été restaurés et présentent donc des résidus de consolidants à leur surface. Les paragraphes suivants sont dédiés aux résultats d'analyse obtenus sur la surface des objets d'Arslan Tash.

\section{Dorure et origine de la couleur des taches pourpres}

Des analyses réalisées en 2006 sur les restes de matériau doré avaient identifié des feuilles d'or pur, mais n'avaient pas pu caractériser les taches pourpres communément considérées comme des restes ou des assiettes de dorure (fig. 7). Un des enjeux de ces analyses était d'identifier la nature de ces taches.

Les analyses microPIXE effectuées sur les objets comportant cette coloration pourpre ont mis en avant la présence non négligeable d'or, allant jusqu'à 3,8 \% massique alors que sur l'ivoire clair limitrophe, les quantités sont en dessous des limites de détection de la méthode. De plus, des analyses en microDRX sur la tache pourpre de l'aile de l'objet AO 11469 ont révélé la présence d'or métallique (fig. 8). Grâce à l'application d'un calcul basé sur l'équation de Scherrer, une taille de particules de l'ordre de $70 \mathrm{~nm}$ peut être déduite de la largeur à mi-hauteur des pics de diffraction de l'or. Ces taches sont par conséquent en rapport direct avec les dorures autrefois présentes sur les pièces et encore visibles sur quelques pièces de la collection.

Lor est par ailleurs connu pour fournir toute une gamme de couleurs du rouge au bleu dans d'autres matériaux sous forme de nanoparticules. En effet, il est utilisé dès l'époque romaine pour créer des verres rouges. Cette technique fut ensuite redécouverte au XvII ${ }^{\mathrm{e}}$ siècle, avec les premiers ouvrages mentionnant le pourpre de Cassius. La couleur est due à des absorptions spécifiques de la lumière visible correspondant à des résonances de modes plasmons de surface à l'interface particule/matrice [8]. 


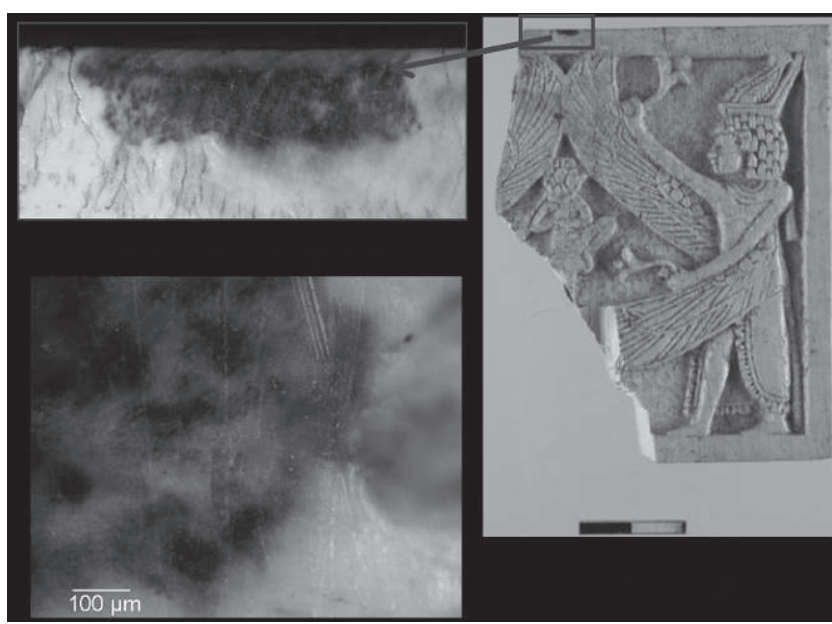

Figure 7 : (Voir planche couleur) Zone pourpre de l'objet AO 11469, observée au microscope optique avec différents grossissements.

Figure 7: (See colour plate) Purple zone of the object AO 11469, observed under the optical microscope optique with different magnifications.

L'hypothèse proposant que la couleur pourpre sur l'ivoire est due au même phénomène a été vérifiée par spectroscopie d'absorption optique dans le visible. Le spectre obtenu sur les restes pourpres de l'objet AO 11469 (fig. 9) montre un profil similaire à celui des glaçures roses à l'or sur des carreaux iraniens du XIX ${ }^{e}$ siècle [9]. En effet, sur les carreaux persans, la couleur est probablement contrôlée lors du processus de fabrication ce qui mène à des tailles de nanoparti- cules relativement homogènes. Pour les taches pourpres, ceci ne semble pas être le cas et reste à vérifier. Ainsi, en observant la zone pourpre de l'objet AO 11469 au microscope optique (fig. 7), il apparaît que la couleur, perçue pourpre à l'œil, n'est en réalité pas une zone uniforme mais correspond à une juxtaposition de zones rouges et pourpres, formées par des groupes de nanoparticules de tailles différentes, les pourpres étant plus petites que les rouges.

La couleur pourpre des ivoires d'Arslan Tash est donc due à des nanoparticules d'or. Il est cependant peu probable qu'il s'agisse d'une préparation déposée lors de la fabrication des objets. Il s'agit de façon plus plausible d'un effet de l'altération de la feuille d'or qui décorait les pièces. Comme pour la préparation du pourpre de Cassius, l'or aura été divisé jusqu’à obtenir des nanoparticules par des composés acides présents soit dans le sol, soit dans le liant utilisé pour coller la feuille d'or.

\section{Traces du bleu-vert}

L'analyse de l'incrustation dans l'œil d'un sphinx criocéphale (AO 11497) en spectroscopie microRaman permet de montrer la présence de bleu égyptien en tant qu'élément de polychromie sur les objets (fig. 10).

\section{Sédiments}

Les restes de sédiments présents sur toutes les œuvres d'Arslan Tash ont été analysés par microPIXE afin de déterminer leur composition élémentaire. En moyenne ont été déterminés (en \% massique) : 47,7 $\mathrm{SiO}_{2}, 15,7 \mathrm{CaO}, 11,2$
Figure 8 : Micro-diffractogramme de rayons X sur la tache pourpre de l'objet AO 11469 montrant par les pics marqués la présence de l'or métallique en plus de ceux de l'apatite.

Figure 8: Micro-X-ray diffraction patterns of the purple dot on the object $A O 11469$ showing peaks characteristic of metallic gold in addition to apatite peaks.

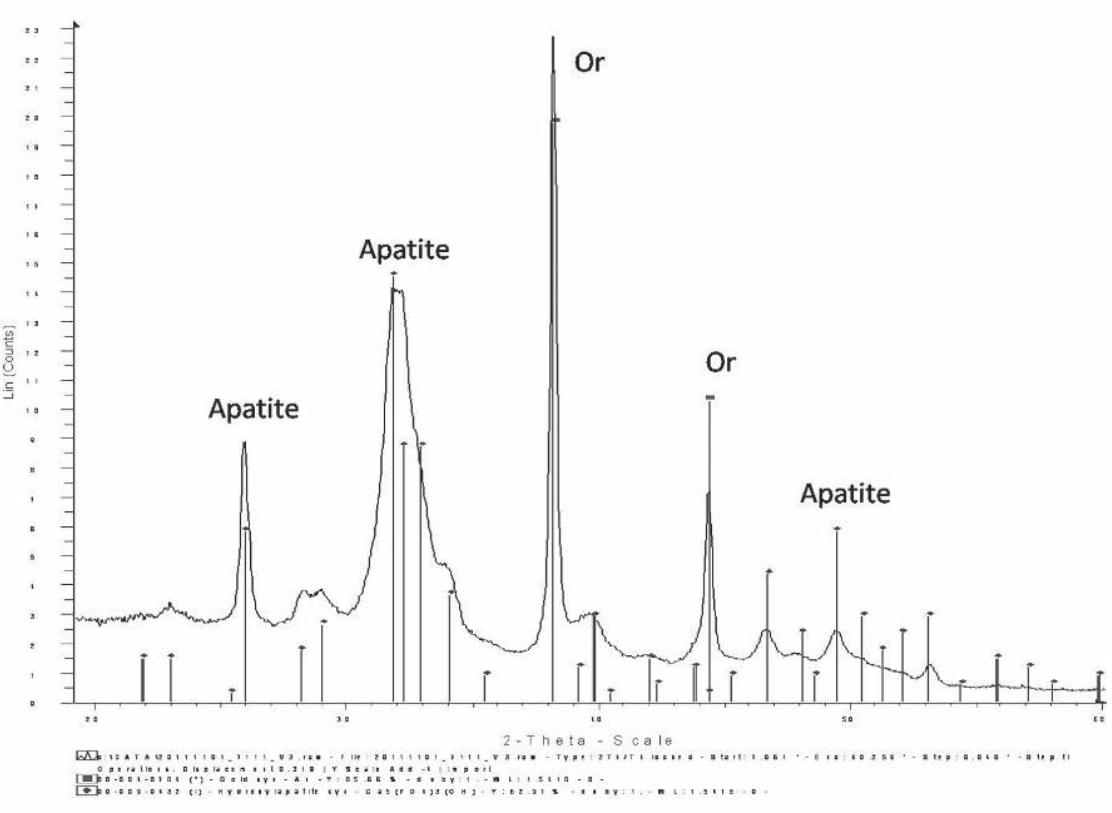

ArcheoSciences, revue d'archéométrie, 35, 2011, p. 283-295 


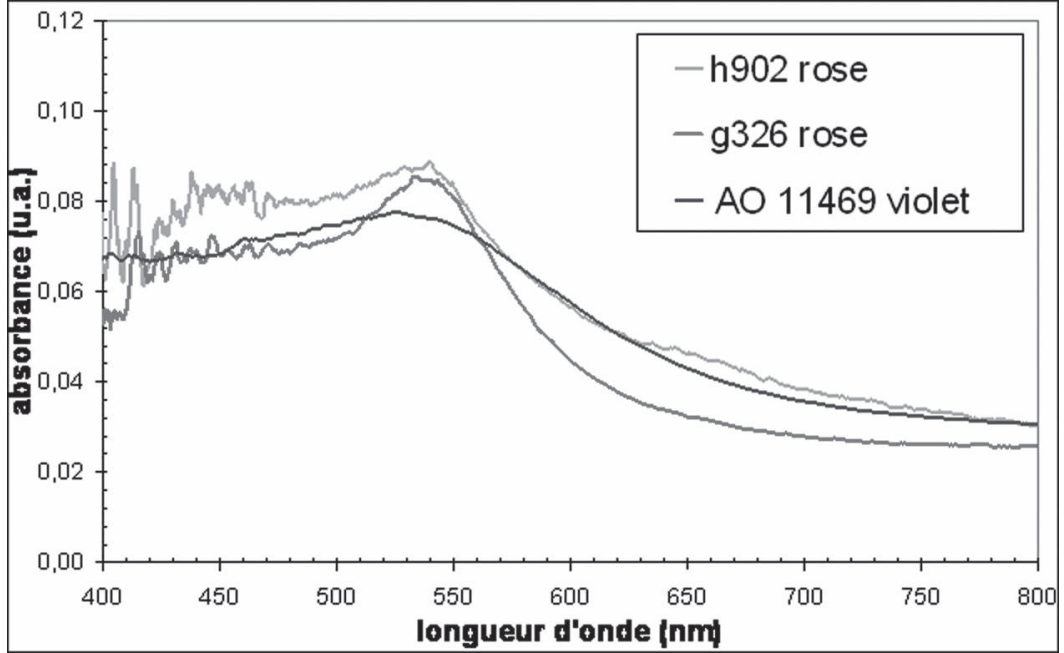

Figure 9 : (Voir planche couleur) Comparaison du spectre d'absorption optique (Visible) de la zone pourpre de l'objet AO 11469 avec ceux des glaçures roses de carreaux persans du XIX ${ }^{e}$ siècle (h902 $=\mathrm{n}^{\circ}$ inv. MAO 902, Musée du Louvre, g326=n inv. 1969-326, Musée des Beaux-Arts, Lyon) (Reiche et al. 2009).

Figure 9: (See colour plate) Comparison of the optical absorption spectra in the visible range of the purple zone of the object $A O 11469$ with those of the rose glazes of Persian tiles of the $19^{\text {th }} c$. (h902= inv. $n^{\circ} M A O$ 902, Musée du Louvre, g326= inv. $n^{\circ} 1969-326$, Musée des Beaux-Arts, Lyon) (Reiche et al., 2009).
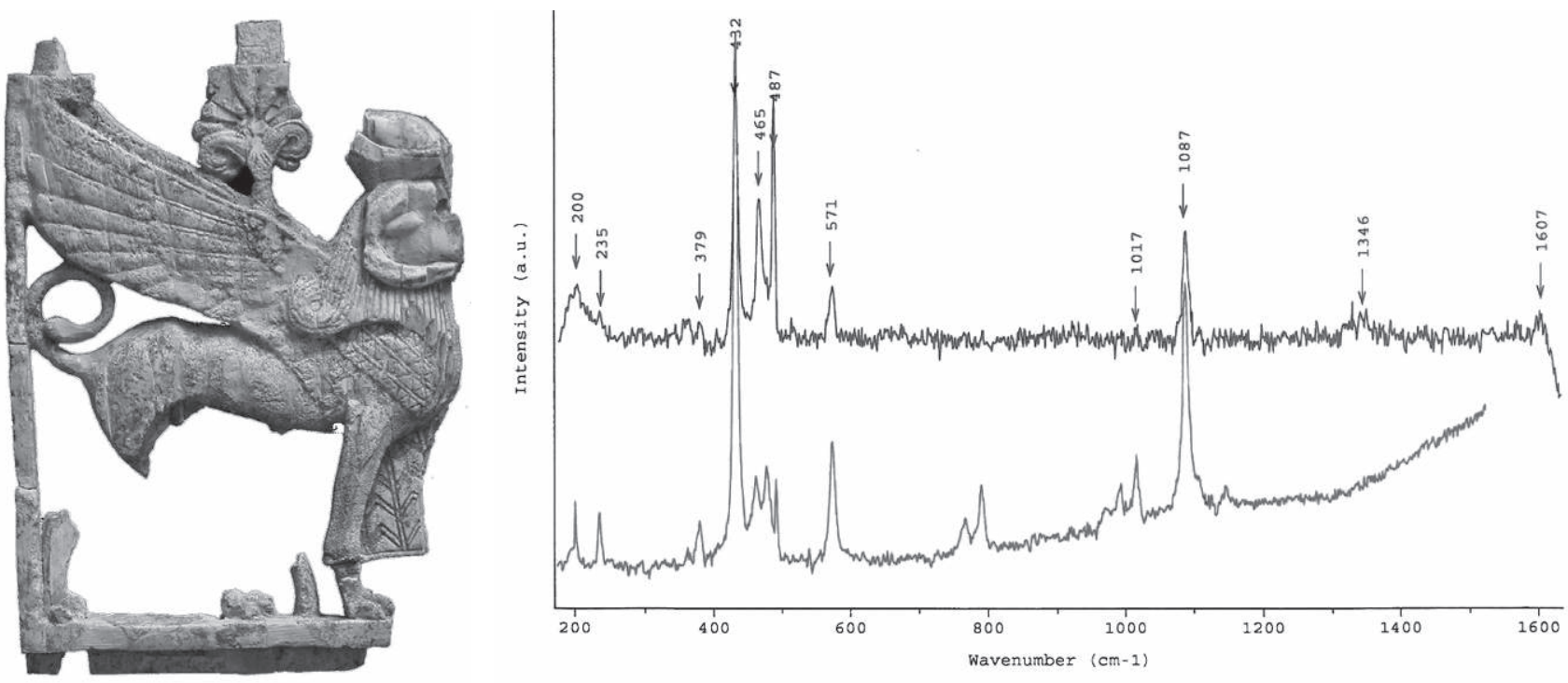

Figure $10:$ a) (Voir planche couleur) Incrustation dans l'œil d'un sphinx criocéphale (à tête de bélier) (AO 11497), b) présence de bleu égyptien démontrée par micro-spectrométrie Raman (spectre de référence en rose).

Figure 10: a) (See colour plate) Inclusion in the eye of the criosphinge (ram-headed sphinxe) (AO 11497), b) presence of Egyptian blue demonstrated by par micro-Raman spectrometry (reference spectrum in pink).

$\mathrm{Al}_{2} \mathrm{O}_{3}, 7,4 \mathrm{FeO}, 4,3 \mathrm{P}_{2} \mathrm{O}_{5}, 3,8 \mathrm{MgO}, 3,7 \mathrm{~K}_{2} \mathrm{O}, 2,6 \mathrm{SO}_{3}, 1,6$ $\mathrm{TiO}_{2}$ et 2,0 d'autres composés.

De plus, des analyses en spectroscopie Raman ont été faites sur les restes de sédiment de l'objet AO 11469 mettant en évidence la présence d'hématite (oxyde de fer) et de noir de carbone.

Afin de mieux caractériser les phases minérales constituant ces sédiments, des analyses en microDRX ont été réalisées sur les restes présents sur l'ensemble des pièces ainsi que sur l'objet AO 11484. Elles ont mis en avant la présence majoritaire de calcite et de quartz, ainsi que des traces d'hématite et d'argiles, probablement un mélange de palygorskite et de clinochlore. Livoire sous-jacent est aussi faiblement visible. Ces données pourraient servir d'indicateurs caractéristiques du site d'Arslan Tash pour différencier les objets qui en proviennent de ceux d'autres sites.

\section{Matériaux de restauration}

Deux types de matériau ont été analysés : le mastic de bouchage beige ou gris et le consolidant de surface présent en grande quantité sur certains objets. Pour les matériaux de bouchage, les analyses microPIXE effectuées sur les objets 
AO 11464, 11466 et 11496a montrent une concentration en oxyde de calcium de près de $92 \%$ massique, ce qui en fait le constituant principal. De plus, des analyses en microDRX effectuées sur le revers des objets AO 11464 et 11466 ont montré que le mastic de bouchage gris utilisé sur de nombreux objets est composé essentiellement de calcite $\left(\mathrm{CaCO}_{3}\right)$.

Presque tous les objets de la collection Arslan Tash portent en surface un consolidant transparent et brillant. Pour les objets $\mathrm{AO} 11466,11467$ et 11469 , où des quantités importantes de ce matériau se sont accumulées dans les reliefs de gravure, des mesures microPIXE ont été réalisées.

Pour l'objet AO 11466, la tache jaune se caractérise par un très fort taux de chlore et est donc de nature organique. Comme il est de plus non-soluble dans l'eau, ceci fait penser pour ces objets à un traitement avec un polymère de type PVC, qui était recommandé dans les années 80 [10] pour préserver les ivoires des problèmes d'humidité. Pour une meilleure identification de ce produit, un micro-prélèvement serait nécessaire afin de pouvoir réaliser des analyses chromatographiques. Toute restauration future devra cependant prendre en compte ce traitement, soit en éliminant cette couche hydrophobe, soit en trouvant des solutions compatibles avec ce produit.

\section{Observations et positionnement des objets dans la défense d'origine}

Pour deux objets tridimensionnels (AO 11484 et 11490), des tomographies $\mathrm{X}$ ont été réalisées afin de mieux identifier l'emplacement de la matière dans la défense d'éléphant à l'origine. Pour la tête de lion (AO 11490), les radiographies et tomographies $\mathrm{X}$ révèlent la forme de la cavité bouchée au fond de la gueule : elles permettent de voir un canal irrégulier transperçant presque l'intégralité de la pièce (fig. 11). Il s'agit très probablement de la pointe de la cavité conique qui existe à la base de la défense et contient la pulpe dentaire pendant la vie de l'éléphant. Cette hypothèse est étayée par l'organisation des fissures du matériau en forme de soleil autour de ce canal. Il apparaît donc que la pièce a été sculptée en utilisant la cavité pulpaire comme ébauche de la gueule, donc au centre, face à la base de la défense (fig. 12a).

Concernant la colonnette (AO 11484), les radiographies et tomographies $\mathrm{X}$ permettent d'étudier le réseau de fissures dans la masse du matériau (fig. 13). Ceci donne des indications sur l'emplacement possible de la pièce dans la défense. L'ivoire d'éléphant grandit cycliquement pendant la vie de l'individu. Par conséquent, sa composition chimique et sa densité varient avec les conditions de vie de l'animal. L'interface entre ces cônes de croissance semble être particulièrement fragile et l'ivoire d'éléphant est connu pour se

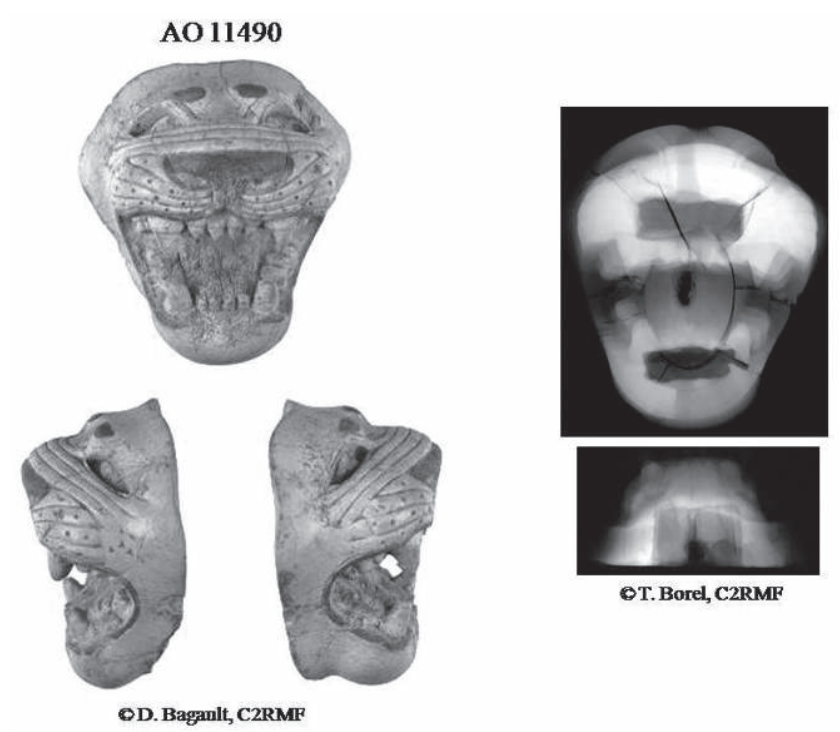

Figure 11 : Tête de lion (AO 11490) et radiographie X. Figure 11: Lion head (AO 11490) and X-ray radiography.

cliver selon ces cônes avec le temps. Ici, la forme et l'orientation des fissures laissent penser que l'objet a été sculpté verticalement, dans la défense, face vers l'extérieur, un peu en décalage par rapport au centre de la défense (fig. 12b).

Un autre faciès encore inexpliqué est révélé par les radiographies et tomographies X. Il s'agit de la présence de trois cavités quasi-sphériques à l'intérieur de la masse de l'ivoire, le long d'une fissure. Nous pouvons supposer que l'objet a été un temps clivé selon cette fissure avant d'être recollé. La forme et la régularité de ces cavités interpellent : elles ne sont pas dues à la cassure ou l'effritement naturel du matériau mais ont certainement été volontairement creusées.

\section{ConClusion}

Par ces analyses physico-chimiques il a été possible de caractériser finement l'état de conservation des pièces d'ivoire provenant du site d'Arslan Tash (Syrie) ainsi que leur polychromie et les traces de dorure. Nous avons notamment élucidé l'origine de la couleur des taches pourpres présentes sur certaines pièces. Il s'agit de l'or sous forme colloïdale. Son processus de formation sur les pièces en ivoire reste cependant à élucider.

Afin de confirmer ou d'enrichir ces résultats une seconde tranche d'analyses d'œuvres du Louvre est prévue en 2011. De plus, le Badisches Landesmuseum de Karlsruhe a accepté qu'une série de pièces de sa collection soient analysées prochainement notamment par fluorescence $\mathrm{X}$ à la source syn- 

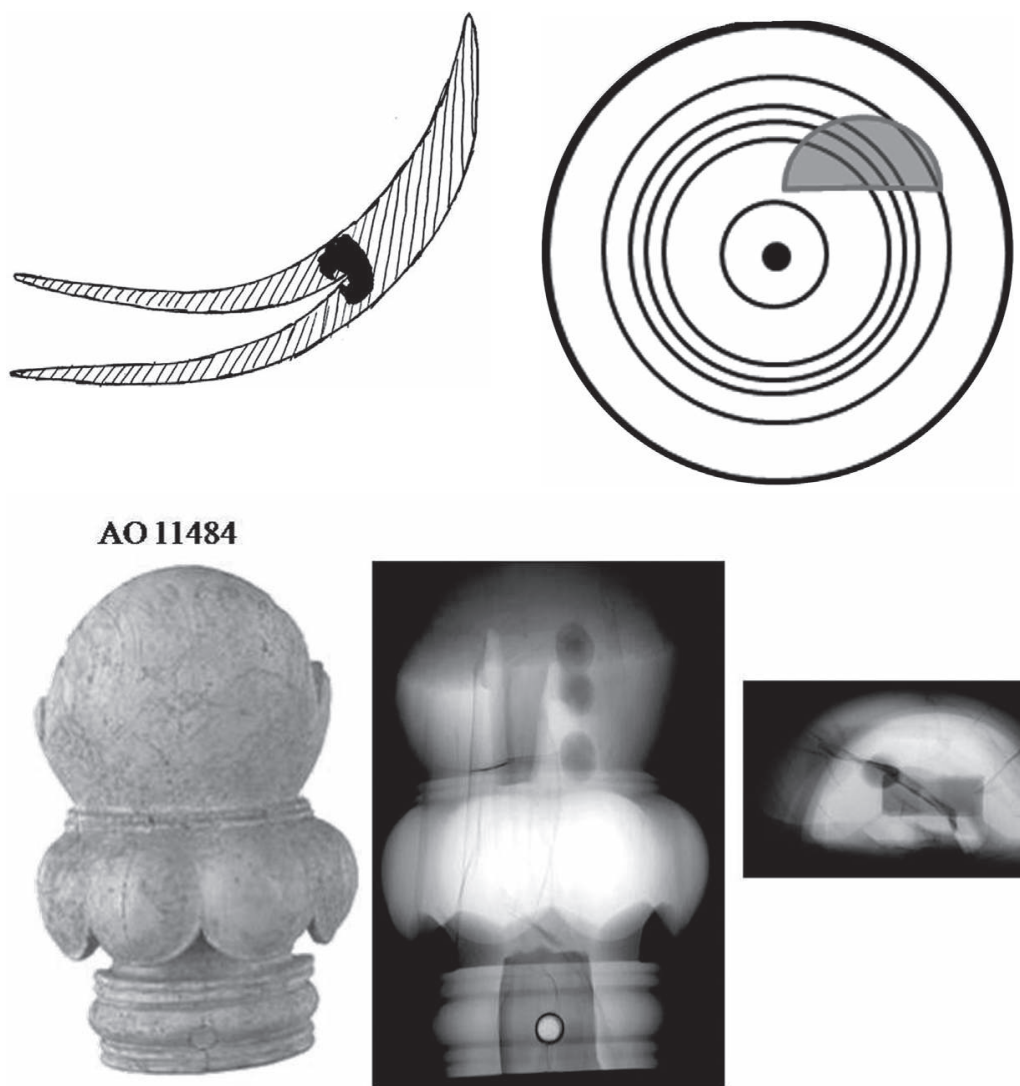

(2) D. Bagault, C2RMF
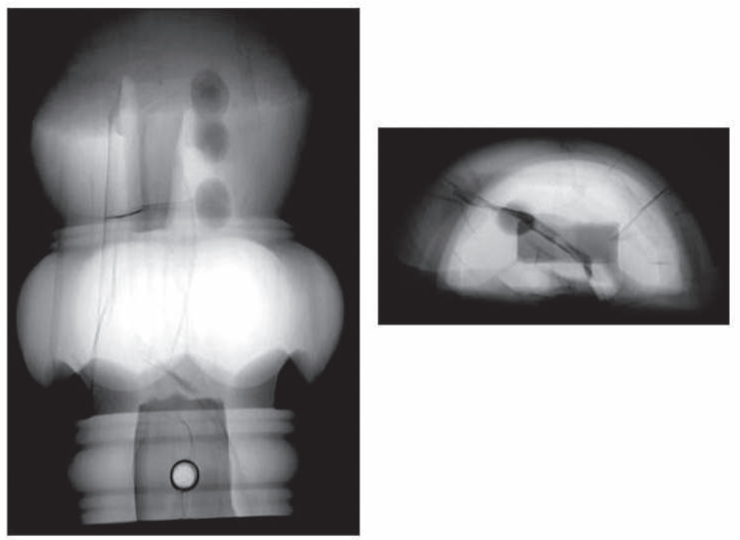

Figure 12 : Propositions de positionnement des objets a) AO 11490 et b) 11484 dans une défense d'éléphant.

Figure 12: Hypotheses for the object positioning a) $A O$ 11490 et b) 11484 within the original elephant tusk used for carving. chrotron ANKA/Karlsruhe Institute of Technology (KIT), Allemagne. Parmi de nombreuses questions, un des points concernant la polychromie est de vérifier si l'aspect " gercé " de la surface de l'ivoire, qui est généralement observé dans les zones teintées en rougeâtre, pourrait indiquer dans certains cas, comme sur le triple cadre des dames à la fenêtre (fig. 14), la présence de couleur à l'origine.

\section{Remerciements}

Nous remercions les collègues du C2RMF : Dounia Large, Katharina Müller, Sandrine Pagès-Camagna, Dominique Bagault, Thierry Borelt, Laurent Pichon et Yvan Coquinot qui ont contribué avec beaucoup d'engagement aux analyses des pièces d'Arslan Tash du Musée du Louvre. Ces travaux ont été réalisés dans le cadre du programme ANR ArBoCo (ANR07-JCJC-0149).

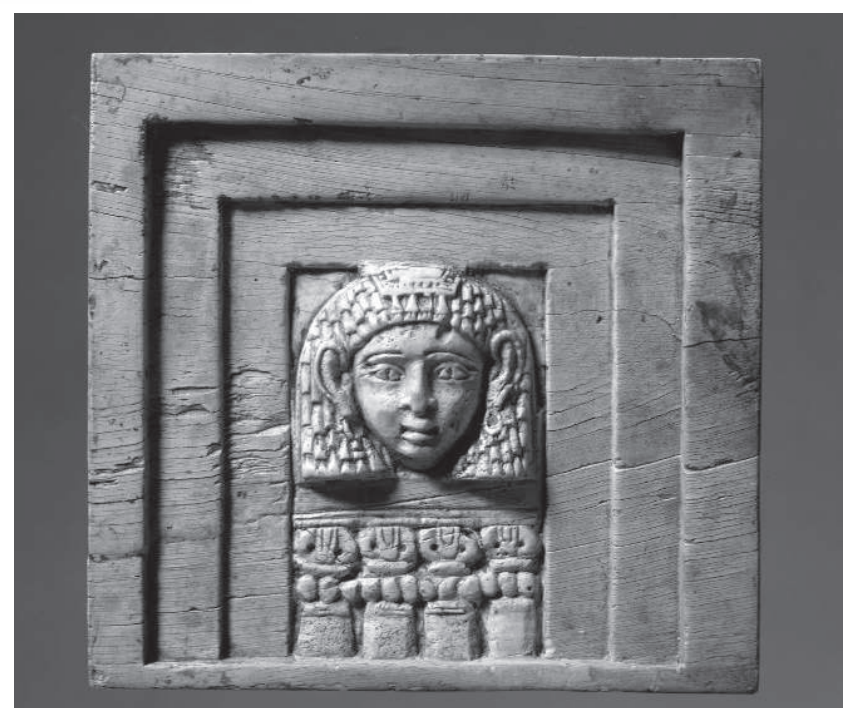

Figure 14 : (Voir planche couleur) Objet "Dame à la fenêtre " (AO 11459), non analysé, montrant l'aspect " gercé » indiquant éventuellement une coloration du triple encadrement, (C) Musée du Louvre/R. Chipault.

Figure 14: (See colour plate) Not analyzed object "Woman at the window" (AO 11459) showing the "chapped " aspect, which indicates possible coloring of the triple frame, (C) Musée du Louvre/R. Chipault. 


\section{Bibliographie}

1. Thureau-Dangin, F. et al., 1931 - Arslan Tash, Paris, Geuthner.

2. Pagès-Camagna, S., 2006 - Plaquettes en ivoire polychromées, Arslan Tash (Syrie), VIIt siècle av. J.-C.., C2RMF rapport interne R 8190, Paris.

3. Calligaro, T. et al., 2002 - Ion beam analysis with external beams: Recent set-up improvements. Nuclear Instruments and Methods in Physics Research B, 188, p. 135-140.

4. Hodean, J. L. et al., 2008 - Dispositif d'analyse d'un échantillon par rayons $X$ comprenant un système analyseur par diffraction filtrant en énergie et en angle, Brevet français $n^{\circ} 0754151$ et US patent application number 12/593, 908, 27 mars 2008.

5. WeIner, S. et O. BAR-Yosef, 1990 - States of Preservation of Bones from Prehistoric Sites in the Near East: A Survey. Journal of Archaeological Science, 17, p. 187-196.
6. WRIGHT, L. E. et H. P. SCHWARCZ, 1996 - Infrared and Isotopic Evidence for Diagenesis of Bone Apatite at Dos Pilas, Guatemala: Palaeodietary Implications. Journal of Archaeological Science, 23, p. $933-944$.

7. Borel, T., 1995 - La radioagraphie des objets d'art, Technè, 2, p. $146-157$.

8. LafaIt, J., 2006 - Coloration des verres par des nanoparticules métalliques. Verre, 12, p. 11-21.

9. ReICHe, I. et al., 2009 - Development of a nondestructive method for underglaze painted tiles - demonstrated by the analysis of Persian objects from the nineteenth century. Analytical and Bioanalytical Chemistry, 393, p. 1025-1041.

10. Lafontaine, R. H. et P. A. Wood, 1982 - The stabilzation of ivory against relative humidity fluctuations. Studies in Conservation, 27, p. 109-117. 